\title{
Psicometría, perfiles
}

y sesgos

El caso del reconocimiento facial

\section{Psychometrics, profiles and biases The case of facial recognition}

\section{Psicometria, perfis e vieses O caso do reconhecimento facial}

DOI: https://www.doi.org/10.18861/ic.2021.16.2.3156

\section{JUANCAMILO GÓMEZ-BARRERA}

jcgomezba@gmail.com - Instituto Colombiano para la Evaluación, Colombia.

ORCID: https://orcid.org/0000-0003-3704-2266.

CÓMO CITAR: Gómez-Barrera, J. (2021). Psicometría, perfiles y sesgos. El caso del reconocimiento facial. InMediaciones de la Comunicación, 16(2), 63-81.DOI: https://www.doi.org/10.18861/ ic.2021.16.2.3156

Fecha de recepción: 12 de marzo de 2021 Fecha de aceptación: 19 de junio de 2021

\section{RESUMEN}

Los estudios psicométricos permiten realizar mediciones basadas en correlaciones de vectores que producen predicciones de rasgos del comportamiento humano. Con la creciente digitalización, la psicometría es usada para diseñar perfiles de los individuos a través de diversos mecanismos: big data, machine mearning, entre otros. Lejosdeser neutrales, estosperfiles están basados en metodologías que producen sesgos que afectan a los individuos perfilados. Así, este artículo muestra cómo la psicometría elabora un perfil-dividuo que genera una reducción de los individuos y produce formas en las que estos tienen que comportarse. Para ello, se ejemplifica con un caso demedición psicométricabasadaen el reconocimiento facial.

PALABRAS CLAVE: psicometría, perfil-dividuo, reconocimiento facial, sesgos.

\section{ABSTRACT}

Psychometric studies allow us measurements based on vector correlations that produce predictions of human behavioral traits. With increasing digitization, psychometrics has been used to design profiles of individuals through various mechanisms: big data, machine learning, among others. Far from being neutral, these profiles are based on methodologies which produce biases that affect the profiled individuals. This article shows how psychometrics elaborates a dividual-profile that generates a reduction of individuals and produces forms that augur the way in which they should behave. Here the conjecture is exemplified from the analysis of a case of psychometric measurement based on facial recognition.

KEYWORDS: psychometrics, profile-dividum, facial recognition, biases. 


\section{RESUMO}

Os estudos psicométricos permitem realizar medições baseadas em correlações de vetores que produzem previsões de traços comportamentais humanos. Com o aumento da digitalização, a psicometria é usada para projetar perfis de indivíduos por meio de vários mecanismos: bigdata, machinelearning, entre outros. Longe de serem neutros, esses perfis baseiam-se em metodologias que produzem vieses que afetam os indivíduos perfilados. Este artigo mostre como a psicometria desenvolve um perfil-dividuo gerando uma redução dos indivíduos e produz formas que pressagiam como eles deveriam se comportar. Aqui a conjectura é exemplificada a partir da análise de um caso de medição psicométrica baseada no reconhecimento facial.

PALAVRAS-CHAVE: psicometria, perfil-dividuo, reconhecimento facial, vieses. 


\section{INTRODUCCIÓN}

Robert William conducía hacia su casa en Detroit, Estados Unidos, cuando fue arrestado por agentes de la Policía. Corría el año 2020, la pandemia azotaba a los barrios marginales en todas las ciudades norteamericanas y se vivía un ambiente de protestas racistas ante el crecimiento de hechos delictivos. Al siguiente día del arresto, un detective se acercó a William con unas fotografías en la mano. Allí se veía el rostro de un afroamericano cometiendo un delito: una cámara de vigilancia lo había captado y un software de reconocimiento facial -Greenlight- había determinado que era William. Para el agente, las fotografías y el veredicto de Greenlight eran suficientes para legalizar la detención. Sin embargo, al ver las fotos, William se percató de algo: "Ese no soy yo", le dijo al detective, sorprendido. Luego, un poco más molesto, inquirió: “¿Crees que todos los hombres negros somos parecidos?”. Y el agente contestó: "El computador dice que eres tú" (Hill, June 24, 2020).

La respuesta que el policía le dio a William muestra cierto halo de mistificación que se le ha dado a la Inteligencia Artificial (IA). Bajo ese manto sagrado, se asume que lo artificial-computacional refiere a una tecnología autónoma de lo humano, e incluso lo desborda, lo supera. De hecho, la IA ha estado sostenida por una metáfora de la caja negra (black box) y sería, por lo tanto, capaz de tomar decisiones por sí misma; es decir, ha sido entendida como una tecnología superior a lo humano, que está por encima de cualquier comprensión y, de alguna manera, se encuentra fuera de control (Lipton, 2016). En oposición a esa postura, autores como Pasquinelli y Joler (May 1,2020) entienden a la IA como un instrumento de conocimiento que permite inferir patrones, características y correlaciones en grandes cantidades de datos. Bajo esa perspectiva, los sistemas de reconocimiento facial, por ejemplo, deben ser comprendidos como instrumentos de medición y percepción: como aparatos que difractan y distorsionan el objeto observado a través de lentes. Solamente así se pueden dimensionar y analizar sus implicancias sociales. En el caso de William, particularmente, se observa que la IA se ha convertido en un "régimen de verdad, de prueba científica, en una normatividad y racionalidad social, la cual toma la forma de una alucinación estadística" (Pasquinelli \& Joler, May 1, 2020,p. 2).

La descripción de Pasquinelli \& Joler (May 1, 2020) puede aplicarse a diversos trabajos psicométricos surgidos a raíz de la creciente acumulación de datos recopilados de bases de datos, redes sociales o plataformas que han estado enfocados en la medición de rasgos de los individuos. La psicometría ha estado enfocada en elaborar mecanismos algorítmicos que permiten predecir comportamientos de los usuarios a partir de tecnologías de rastreo, análisis y producción de perfiles basados en tecnologías de aprendizaje automatizado (o machine learning, en inglés). Si bien la psicometría es anterior a Internet, con la emergencia de las plataformas y bases de datos se han podido desarrollar 
mecanismos que pueden predecir atributos personales a partir de la medición del lenguaje utilizado por los usuarios, así como sus gustos e intereses (GómezBarrera, 2020). Es decir, la psicometría se ha convertido en un instrumento de conocimiento, así como en una suerte de pauta de normatividad racional sobre los objetos -que son los humanos-, los cuales son medidos y evaluados, y sobre la cual se pueden tomar decisiones de distinto tipo. Un caso que ejemplifica ese procedimiento se puede encontrar en el reconocimiento facial.

Lejos de ser un sistema objetivo de identificación de rostros -o características propias de las personas-, las tecnologías de reconocimiento facial tienen como objetivo producir perfiles. Esto quiere decir que a través de análisis psicométricos sobre rasgos físicos y tecnologías de inferencia se formulan evaluaciones que permiten determinar aspectos relacionados con el comportamiento de los individuos. Los desarrollos contemporáneos del reconocimiento facial están abocados a tomar una serie de datos de fotografías de personas, medirlas y analizarlas mediante técnicas estadísticas, compararlas con patrones previos y producir perfiles que -de acuerdo con esta mirada- permiten determinar aspectos relacionados con diversos aspectos comola sexualidad, las tendencias violentas y la inteligencia.

En relación con lo anterior, el reconocimiento facial no es ajeno a ser una fuente de recursos para mediry evaluar diversos aspectos del comportamiento. Algunos de los más recientes estudios han estado enfocados en la identificación de la sexualidad, la edad y los estados de ánimo a partir de la medición biométrica. Wang y Kosinski (2018) mostraron cómo se podían identificar aspectos relacionados con la sexualidad: diseñaron un algoritmo -sostenido en un contraste mediante una regresión estadística y una medición psicométrica- que les permitió identificar, a través de una serie de fotografías, si los hombres (el 81\% de los casos contrastados, y las mujeres: el 71\% de los casos) eran heterosexuales. Este algoritmo se basó en el análisis de aspectos del rostro como la nariz o estilos de peinado, que responden a teorías que sustentan que las personas homosexuales tienden a poseer una fisiología facial atípica, expresiones diferenciales en poses y de cuidado personal distintivos. Estas marcas diferenciales les permitieron a Wang y Kosinski (2018) desarrollar un algoritmo que pretende predecir la sexualidad de un individuo.

Otros estudios se han enfocado en poder determinar rasgos de la personalidad de los individuos a partir de las fotos de perfil de redes sociales como Facebook. Así, Segalin y otros autores (2017) desarrollaron un sistema automático de reconocimiento de rasgos de personalidad a partir de patrones del comportamiento como la extraversión y el neuroticismo. En ese estudio, determinaron que las personas que son más extrovertidas tienden a usar fotos de perfil más coloridas; de igual manera, quienes tienen un carácter más agradable usan diferentes escenarios y publican fotografías con diversas personas, demostrando con ello un carácter sociable. Por su parte, las personas 
que consideran que tienen un carácter más neurótico tienden a usar fotos de perfil tomadas en locaciones cerradas y oscuras, a ocultar el rostro o publicar fotografías que no son propias. Bajo esos parámetros, los autores afirman poder determinar un sistema de clasificación automatizada de la personalidad (Segalin et al., 2017).

Ahora bien, este tipo de desarrollos psicométricos sobre reconocimiento facial tiene alcances que desbordan las plataformas en las que están basadas. Por un lado, generan problemáticas en torno a la vigilancia y, por otro, pueden desencadenar sesgos sociales que afectan a los individuos: por ejemplo, si con base en esos análisis se toman decisiones financieras o policiales, como el caso de William antes descrito. De allí que en este artículo se detalle un ejemplo de cómo se diseñan sistemas de medición del comportamiento a partir de evaluaciones psicométricas del rostro, lo cual permite evidenciar que las tecnologías de perfil están relacionadas con metodologías que implican, en sí mismas, sesgos que afectan directamente a los individuos. Gran parte de esta afectación puede comprenderse bajo el concepto de perfil-dividuo, el cual expresa la tendencia a reducir a los individuos y producir formas en las que estos tienen que comportarse. Para explicar esta afectación, se hace uso dela teoría de la gubernamentalidad algorítmica, la cual permite formular las implicaciones inmediatas que se despliegan con el uso de este tipo de tecnologías digitales, y se trabaja sobre un caso específico de reconocimiento facial relacionado con la identificación de inclinaciones políticas de los individuos.

\section{MEDICIÓN Y CORRELACIÓN: BAJO EL MANTO DE LA PSICOMETRÍA}

Uno de los fundamentos de la psicometría es la medición. En términos generales, la idea de medir refiere al establecimiento de propiedades de objetos o individuos a través de una descripción en términos numéricos (Geisinger, 2013). Aquello medible pueden ser rasgos observables de manera directa, como la estatura de una persona, pero también pueden ser rasgos no observables, como la inteligencia o las capacidades físicas. En principio, la psicometría se enfoca en la medición de rasgos no observables (más conocidos como latentes), de acuerdo con escalas, herramientas de medición o criterios previos.

La medición fue definida por Stevens (1946) como la asignación de números a objetos o acontecimientos con base en productos. De una medición se pueden obtener escalas de diferente tipo, por lo que no se trata de un proceso de mera cuantificación, sino que es un ejercicio que a su vez valida las técnicas estadísticas utilizadas. En el desarrollo de la psicometría como ciencia, las técnicas implementadas estuvieron influenciadas por soportes estadísticos, en especial, de técnicas como el Múltiple Factor Analysis, que funciona como una herramienta que permite generar validez de los instrumentos de medición que usa la psicometría (Messik, 1989). 
Partiendo de esos principios, una primera definición de la psicometría está relacionada con el uso de una serie de instrumentos de medición, espacialmente de pruebas (Martínez, Hernández \& Hernández, 2006). Por prueba, o test, puede entenderse una multiplicidad de instrumentos como evaluaciones, ensayos, encuestas, etc., destinados a la medición psicológica. Una segunda definición de psicometría está relacionada con la evaluación de fenómenos psicológicos (Rust \& Golombok, 2009). Esta definición, apegada a la etimología de la palabra, implica que la psicometría desarrolla medidas que deben ser científicas y aplicables a la personalidad, lo cual conlleva que las mediciones deban tener un carácter estandarizando y cuantitativo, es decir, que funcione sobre resultados aplicables a una población amplia.

A la hora de tensionar esta idea centrada en la estandarización, recientes definiciones de psicometría plantean que se trata más bien de una teoría y una práctica que miden factores psicológicos que tienen el carácter de ser medibles (Holden, 2000). De este modo, la psicometría ha sido asociada al desarrollo de unas técnicas y métodos relacionados con la medición de rasgos psicológicos de acuerdo con métricas y escalas (Muñiz, 2003). Cabe indicar que esta medición busca establecer inferencias a partir de los datos recogidos por los instrumentos de medición (Jones \& Thissen, 2007). Por tanto, una definición general de la psicometría gira en torno al uso de teorías, métodos y técnicas de instrumentos de medición enfocados en fenómenos psicológicos. El objetivo de esas mediciones está relacionado, en consecuencia, con poder clasificar, predecir y describir rasgos latentes con miras de poder tomar decisiones sobre los individuos medidos.

Vale recordar que la historia de la psicometría es un entrecruce de desarrollos educativos y de mediciones de la inteligencia, así como la puesta operativa de herramientas y pruebas para servicios militares. En ese sentido, los trabajos de Alfred Binet sobre mediciones de aptitudes en estudiantes fueron adoptados, luego, por Lewis Terman, quien en las primeras décadas del siglo XX desarrolló el Stanford Binet, un estudio que permitió establecer estándares para la aplicación y la construcción de pruebas psicométricas que permitieran determinar la medida de inteligencia de un individuo. Este estudio fue utilizado por el ejército norteamericano en la Primera Guerra Mundial, gracias a lo cual la psicometría tuvo el respaldo económico y científico que precisó para su posterior desarrollo. De hecho, la psicometría fue una ciencia usada en ámbitos militares para la selección de reclutas y, en menor medida, a espacios educativos; esto no la salvó de estar vinculada a prácticas discriminatorias.

Las tecnologías digitales y todo lo relacionado a la big data, el aprendizaje automatizado, las plataformas de Internet, entre otros fenómenos, han venido incrementando el uso de mediciones psicométricas y, a la par, han generado que las mediciones integren nuevas herramientas y teorías. La psicometría, en particular, ha aprovechado las grandes cantidades de datos almacenados para 
potenciar los análisis y las mediciones en diversos aspectos del comportamiento humano que antes eran poco explorados. Uno de sus campos de acción ha sido el de la publicidad: a través de diversas mediciones, hoy es posible producir propagandas personalizadas y dirigidas (Rust, Kosinski \&Stillwell, 2021). Cabe indicar que, de manera análoga, los estudios psicométricos han tenido una gran participación en el diseño de diversas plataformas de Internet, en especial las redes sociales. Así, diversos estudios han estado encargados de desarrollar funciones de herramientas como el botón "Like" de Facebook o relacionadas con la comprensión de fenómenos como la propagación de contenidos. Es decir, existe una fuerte demanda del uso de análisis psicométricos enfocados en la predicción de comportamientos que sirvan para el mercado (Redacción MIT Technology Review, 1 marzo de 2021).

Asimismo, una de las ventajas que ha propiciado que la psicometría pueda trabajar con grandes cantidades de datos es el hecho de que haya desarrollado métodos y teorías del uso de datos previos a la existencia de la big data. Uno de los primeros casos donde se usaron análisis psicométricos con grandes cantidades de datos fue en el proyecto Army Alpha IQ Test, diseñado por Robert Yerkes, que utilizó el Ejército norteamericano en la Primera Guerra Mundial (Stone \& Yerkes, 1920). De igual manera, las herramientas de medición que usaron algoritmos fueron desarrolladas en la neuropsicología, especialmente con el planteamiento de las redes neurológicas, proceso que fue previo al aprovechamiento de la IA como herramienta de medición (Rust, Kosinski \& Stillwell, 2021). Con esto, lo que se observa es que la psicometría ya contaba con una batería de herramientas, teorías y métodos que le permitieron converger con facilidad en el análisis de tecnologías digitales.

El aprendizaje automatizado es una de las herramientas que más ha aprovechado la psicometría para el análisis en entornos digitales. Los primeros estudios psicométricos que trabajaron con aprendizaje automatizado fueron los de Donald Hebb, en 1940, sobre el uso de redes neuronales como mecanismos de aprendizaje. Junto con la teoría de Alan Turing, estas nociones brindarían las bases para el desarrollo de la IA. A grandes rasgos, lo que trabajó Hebb era la posibilidad de que un sistema computacional pudiera aprender a partir de los datos con que era alimentado; es decir, las redes neuronales son una serie de nodos que un algoritmo es capaz de relacionar, analizar y producir conexiones. El resultado de este entrecruce de datos es una inferencia de elementos que en la entrada de información no estaban asociados -a esto, en otros términos, puede denominárselo establecimiento de patrones-.Este tipo de correlaciones es usado por los estudios psicométricos, ya que permiten deducir regularidades en una inmensa masa de datos, que a su vez permiten o bien clasificar o bien inferir predicciones de patrones (Bishop, 2006).

El desarrollo del aprendizaje automatizado, centrado en la necesidad de correlacionar inmensas masas de datos, hallevado a la psicometría a usar herra- 
mientas de la estadística moderna, en especial, las redes bayesianas. A grandes rasgos, una red bayesiana es un gráfico que permite relacionar variables aleatorias que en principio pueden ser observables, latentes o hipotéticas. Se trata de una herramienta que permite visualizar la unión probabilística de variables en un modelo complejo, entendido a través de una relación teórica o empírica (Pearl, 1988). Esta representación gráfica permite un punto de contacto entre las grandes cantidades de datos y los análisis psicométricos, enfocados en construir el modelo matemático y delimitar las inferencias establecidas. Por tanto, la idea principal de las redes bayesianas es la de proveer una distribución probabilística que permita correlacionar variables diversas para formular un conocimiento específico sobre un objeto o evento determinado. Es decir, las redes bayesianas grafican -o ilustran - la probabilidad de distribución (o cercanía) y correlación de variables (o aspectos) sobre las cuales se puede extraer información (Gelman, 2013).

Al posibilitar correlacionar aspectos de masas de datos y producir predicciones e inferencias, las redes bayesianas son actualmente usadas en mediciones psicométricas que usan big data (Rust, Kosinski \& Stillwell, 2021), principalmente, porque permiten relacionar una manifestación observable con un rasgo latente, no observable; por ejemplo, pueden correlacionar el uso frecuente de la palabra amor (lo observable) que tiene un individuo con un comportamiento afectuoso o un sentimiento (algo no observable); esto es, inferir que si alguien usa mucho esa palabra es afectuoso. De hecho, entre más datos se tengan de un individuo, mejores correlaciones se van a poder efectuar, y, a su vez, más inferencias se podrán deducir del comportamiento. De allí que las redes sociales digitales, en parte, estén avocadas a la captación de datos de cualquier tipo: desde físicos, geográficos hasta comportamentales. Al trabajar con una gran cantidad de datos, las redes bayesianas permiten correlacionar múltiples y diferentes aspectos observables de un individuo, como el desplazamiento por una ciudad, con datos sobre lo que ese individuo compra y lo que ve en sistemas de streaming. Esta correlación es usada por la psicometría para determinar, por ejemplo, si ese individuo tiene cierta inclinación sexual o si tiene cierta tendencia ideológica; es decir, formular y plantear rasgos no observables. Es decir, se pueden correlacionar los lugares por los que se desplaza un individuo con las series audiovisuales que ve y lo que escribe, y de esa manera inferir, por caso, las tendencias políticas: por caso, un estudio psicométrico podría establecer que alguien que frecuente los centros comerciales, escuche música pop y escriba de manera reiterada la palabra "romántico" en sus conversaciones tiene una tendencia política conservadora.

Bajo la lógica de las redes bayesianas, la correlación que se hace entre aspectos observables para determinar rasgos latentes (no observables) parte del hecho de que se considera verdadera toda relación entre variables que arrojen un resultado válido: por ejemplo, determinar una tendencia política específica 
en tanto se establezca una correlación entre ir a centros comerciales y escribir la palabra romántico. En esa medida, las correlaciones se constituyen en un régimen de verdad, dado que permitirían, según esta lógica, desarrollar inferencias y demostrar hipótesis. Dehecho, toda correlación establecida estadísticamente se vuelve significante: una palabra con una posición geográfica puede relacionarse con estados de ánimo, o la forma de las cuencas de los ojos con inclinaciones depresivas (Mislevy \& Gitomer, 1996; Rust, Kosinski \& Stillwell, 2021). La consideración de toda correlación como un hecho verídico supone un problema epistemológico que envuelve problemas relacionados con cómo se construyen formas de pensary razonar (Markman, 1998); dehecho, las bases que sustentan la correlación como una forma de razonamiento válido para la ciencia moderna están enraizadas en formas de representación científica (Suárez, 2004), como en la genética (Stewart \& Hafner, 1995) o en la arquitectura (Brand, 1994). Si bien no es la intención de este artículo abordar ese problema científico que supone la correlación, basta con enunciar que ha sido determinada como una forma válida y verídica de comprobación de cualquier conjetura; esto es, es una forma de razonamiento sobre el cual se pueden producir inferencias (Sowyer, 1991; Sampson, 1981).

En síntesis, la correlación de variables, vía redes bayesianas o cualquier función estadística, es el principio de las mediciones psicométricas basadas en el análisis de grandes cantidades de datos. El pilar de esta correlación es la extracción de datos dispares -y de diversa naturaleza- que puedan ser objeto de una hipótesis asociada a un comportamiento psicológico de aquello que se quiera observar. En esa medida, lo que se procura es el establecimiento de patrones clasificatorios o predictivos sobre los individuos, con base en datos asociados a rasgos previos: es decir, medibles. Esa operación es la que permite entender que la psicometría se encarga de producir perfiles de los individuos a partir de big data. Y a partir de esos perfiles toman decisiones financieras, políticas, educativas, de salud y otras. A continuación, se mostrará un ejemplo de esto con el reconocimiento facial.

\section{3. ¿QUÉ DICE MI ROSTRO DE MÍ?}

Como se ha planteado, a través del uso de datos almacenados en redes sociales y sistemas biométricos se han diseñado mecanismos de predicción del comportamiento y de conductas relacionadas con la sexualidad, los estados de ánimo, los consumos. De esta manera, se han desarrollado evaluaciones psicométricas que han permitido diseñar algorítmicos capaces de identificar las inclinaciones o las afiliaciones políticas de los individuos a través del reconocimiento facial.En ese marco, se presentará un estudio desarrollado por Kosinski (2021) que busca demostrar que las inclinaciones políticas, entre liberales y conservadoras, en tres países -Canadá, Estados Unidos y Reino Unido- pueden ser inferidas mediante el reconocimiento facial. 
La identificación de características del comportamiento a través del rostro ha sido estipulada por diversas investigaciones psicométricas (Zebrowitz, 1997). Muchos aspectos no verbales pueden ser comunicados e identificados a través del rostro: estados de ánimo, disposiciones, rasgos identitarios, entre otros. De hecho, se ha investigado cómo el rostro puede estar relacionado con el éxito a la hora de obtener un empleo, por el simple de hecho de las impresiones que se pueden generar de manera instantánea (Rule \& Ambady, 2008; Roberts \& Bruce, 1988). La sexualidad, por su parte, ha sido uno de los aspectos que se ha demostrado ser más fácilmente identificable por otras personas de acuerdo con ciertos rasgos específicos del rostro o la composición de las fotografías (Rule \& Ambady, 2008). Sin embargo, un rasgo que ha llamado la atención de los estudios psicométricos relacionados con el reconocimiento de comportamientos a partir del rostro está relacionado con las inclinaciones políticas. A pesar de que ha sido demostrado que el éxito de victoria de un candidato depende, también, de cómo se percibe en fotografías (Ballew \& Todorov, 2007), los estudios de la identificación de inclinaciones políticas han sido más relativos.

Una primera aproximación direccionada a demostrar si las inclinaciones políticas podían ser identificadas a partir de los rostros fue llevada a cabo por Rule y Ambady (2010). En su estudio, estos investigadores plantearon que las afinidades políticas podían ser inferidas con precisión a partir de ciertos rasgos del rostro de las personas. Si bien se trató de un estudio que buscaba demostrar si las personas eran republicanas o demócratas, sirvió de base para iniciar estudios sobre los rasgos particulares que podía tener un rostro de acuerdo con sus ideales políticos. A partir de la identificación de estos rasgos, podía iniciarse un trabajo de programación para que el reconocimiento facial mostrara la efectividad de la hipótesis. En tal sentido, el trabajo de Rule y Ambady (2010) estableció algunos parámetros útiles en los estudios psicométrico, dado que permitió ampliar el conocimiento de aquellos elementos o variables que determinan las características especiales de los rostros en torno a un comportamiento ideológico: se iniciaron investigaciones que buscaban precisar y delimitar los rasgos específicos que podían evidenciar y efectuar una medición de las tendencias políticas.

Otro trabajo de referencia en estudios psicométricos centrado en la posibilidad de predecir un comportamiento político fue el que realizaron Carpinella y Johnson (2013). Para ellos, la predicción de la afiliación política de un rostro depende del género. Así, en un primer experimento plantearon que la identificación de los rostros republicanos era mucho más precisa en mujeres, porque sus rostros respondían con más precisión a las características que se asumían típicas de alguien con esas filiaciones políticas. En el caso de los demócratas, demostraron que los rostros masculinos tenían una afinidad más cercana con los rasgos prototípicos. 
Tanto el estudio de Rule y Ambady (2010) como el de Carpinella y Johnson (2013) han servido de base para la identificación de aquellas variables que permitirían predecir las filiaciones políticas de un individuo (o el rasgo latente). Con base en esas variables, se han podido programar algoritmos de reconocimiento facial que han permitido efectuar una detección automatizada de los rostros, a partir de herramientas estadísticas como la correlación y las redes bayesianas. Tal como se mencionó, uno de los estudios de caso más significativo ha sido efectuado por Kosinski (2021). Con base en más de un millón de fotografías de perfil de usuarios obtenidas de la red social Facebook, este estudio psicométrico determinó una tecnología que era capaz de determinar las orientaciones políticas de los rostros -en un rango de liberales y conservadores-. Las fotos de perfil, tomadas de Canadá, Estados Unidos y Reino Unido, fueron procesadas con base en una base de datos denominada VGGFace2, la cual permite recopilar y clasificar una serie de rasgos específicos de los rostros, entre poses, formas, colores, edades, etc. (Cao, et al., 2018). De igual manera, el estudio adoptó algoritmos dispuestos para el procesamiento de los rasgos específicos en Face++, una de las tecnologías de reconocimiento facial más usadas a nivel mundial'.

El estudio de Kosinski (2021) partió de una base de datos de fotografías que fue convertida en elementos descriptivos; es decir, se tomaron las muestras de fotos de perfil y se analizaron a través de vectores que permitían reducir, o modelar, los rostros de ciertos núcleos de características. Si se quiere, este procedimiento es una correlación entre rasgos detectados a través de una red bayesiana que permitió deducir características específicas de los rostros. Estos rasgos son abstraídos con la intención de identificar la correspondencia entre dos fotografías. Como forma de contraste, se efectuó una prueba a los individuos de quienes se extrajo la foto de perfil para luego comparar si el análisis correspondía con la inclinación política que habían reportado.

Algunos de los rasgos faciales identificados por Kosinski (2021) como determinantes de inclinaciones políticas están relacionados con el color de la piel, la edad y el género. Así, se pudo determinar que los hombres blancos y de edades superiores a los 40 años tienden a ser más conservadores. Adicionalmente, si bien existen estudios psicométricos anteriores que han determinado una correlación de la escrupolosidad, que encierra rasgos como la organización y la eficiencia con tendencias políticas conservadoras (Sibley, Osborne \& Duckitt, 2013), Kosinski (2021) determinó que rasgos de comportamiento como la apertura a la experiencia y la extraversión (comportamientos como la curiosidad y la sociabilidad) estaban relacionadas con filiaciones políticas más liberales.

La identificación de características del rostro con las tendencias política correlacionó cuatro grandes aspectos de las fotos de perfil: pose de la cabeza

1 Al respecto, véase: https://www.faceplusplus.com/ 
(entre rodada, si tiene un cabeceo hacia algún lado, si hace algún tipo de guiño), expresión de alguna emotividad (si expresa enfado, tristeza, sorpresa, miedo), si usa lentes o gafas (de sol o correctivas) y el corte de cabello (Kosinski, 2021). Los resultados de esta correlación, entre la manifestación de una afinidad política con los rasgos de las fotos de perfil, determinó que la orientación de la cabeza y la expresión evocada de los usuarios predice las tendencias políticas en un 60 $\%$. Por ejemplo, se demostró que los liberales tienden a mirar más fijamente a la cámara, además de que tienden a mostrar sorpresa y muy pocas probabilidades de mostrar disgusto. Por su parte, la forma del peinado y aspectos relacionados con gafas correctivas tuvieron una predicción del $52 \%$. Sin embargo, cuando se combinaron los aspectos se pudieron determinar las tendencias políticas con un $73 \%$ de efectividad.

Peseal hecho de quelos porcentajes de asertividad delaidentificación facial no son concluyentes -lo cual es obviamente razonable-, el estudio de Kosinski abre la posibilidad de que se puedan seguir analizando y correlacionando otro tipo de rasgos observables de los rostros y del cuerpo que permitan generar mejores predicciones, ya que como se indicó en el apartado anterior, las redes bayesianas funcionan mejor si cuentan con una gran cantidad de masas de datos. En tal sentido, una de las recomendaciones es poder efectuar una biometría de la posición de los brazos y del cuerpo entero para seguir haciendo más preciso el perfilado de los individuos. El método y procedimiento de esa correlación sigue siendola misma: intentar, mediante redes bayesianas, asociar gestos, formas de ubicación de la cabeza y maneras de mirar a la cámara, entre otras posturas, con las tendencias políticas de los individuos.

En ese marco, el alcance de este tipo de estudios implica problemas de afectación de la privacidad y limitaciones o sesgos que están dados por el modo de perfilar y clasificar a los individuos de acuerdo a dos tendencias políticas. De allí que, entre otras cuestiones o debates suscitados, surge una pregunta acerca de las implicaciones que estos modos de abordaje y perfilamiento tienen en los individuos. Más allá de que tomen sin consentimiento las fotos de los usuarios de plataformas y redes sociales, cabe preguntarse por las consecuencias de perfilar y vender los resultados obtenidos a instituciones estatales, de policía, crediticios o educativos, cuyo manejo de los datos es difícil de mensurar y muchas veces llevan, por ejemplo -y tal lo descrito en relación a lo sucedido con William-, a escenarios de abuso, represión o violencia política amparada en el uso de la IA.

\section{EL PERFILAD0: VIDAS PARALELAS}

Las sociedades de control, caracterización realizada por Deleuze (1999), ayudan a comprender gran parte del fenómeno expuesto: a partir de una gran base de datos se diseñan métodos de perfilado psicométrico que permiten tomar 
decisiones sobre los individuos. Una de las formulaciones que encontramos en su ensayo Posdata a las sociedades de control tiene que ver con el hecho de que los individuos se convierten en dividuos. Bajo este concepto, se ha entendido que sobre el individuo se ejerce una operación quelo duplica (Rodríguez, 2019). Estosperfiles se elaboran a partir de bases de datosy, según lo que se expuso hasta acá, a partir de operaciones, como las investigaciones psicométricas, que han tornado aún más sofisticados los mecanismos, entre otros, de control.

Dicho perfil está encaminado a constituir una representación del individuo que permita comprenderlo, clasificarlo y predecirlo: como lo mostré en este caso, se busca comprender sus inclinaciones políticas de una manera automatizada, de forma casi instantánea. Sin embargo, ese perfil-dividual no debe ser comprendido como una duplicación, sino una multiplicación de datos que, más tarde, "encontrará al individuo del cual supuestamente provienen y al cual supuestamente vuelven" (Rodríguez, 2019, p. 452). En esa medida, puede considerarse que el perfil construido bajo teorías y metodologías estadísticas y psicométricas puede ser pensado como un dividuo, cuyos efectos replantean, por un lado, las relaciones que los individuos guardan con los perfiles y, por otro, las relaciones que los individuos tienen consigo mismos.

Bajo esa misma perspectiva de las implicaciones del perfilado, Berns y Rouvroy (2016) plantean que la construcción de perfiles digitales se desarrolla de manera arbitraria con respecto a los individuos. Esto quiere decir que la recolección de información de las bases de datos, el procesamiento y la formulación de resultados algorítmicos contraen sesgos que afectan directamente a los individuos perfilados. De hecho, estos autores indican que la recolección de los datos se efectúa de manera en la que no se consideran los contextos o las situaciones en las que el dato se produce. Por ejemplo, en el perfilado de un usuario no se consideran como vectores las circunstancias bajo las cuales se obtienen los datos: es decir, para continuar con el caso del reconocimiento facial, no se plantea un vector en el cual un individuo tenga, por ejemplo, que usar lentes, hecho que puede definirlo como conservador. Todos estos factores circunstanciales en los que se producen esas huellas digitales no son considerados nunca en las mediciones psicométricas, debido a que esto invalidaría e impediría una abstracción que es necesaria para la formulación de una correlación de variables.

Adicionalmente, la construcción de perfiles tiene un efecto inmediato en los individuos, en la medida en que su construcción y delimitación afecta directamente su vida cotidiana. De acuerdo con Berns y Rouvroy (2016), el perfil-dividual limita las acciones y el ambiente a una serie de variables consideradas y específicas sobre las que se entiende la realidad. Así, por ejemplo, si se determina que una mirada sonriente corresponde más a un comportamiento liberal, y alguien quisiera encajar en esa categoría, deberá posar de esa manera. Esto implica que se producen normatividades inducidas a partir de esos perfiles 
sobre las cuales se tiende a modular los comportamientos de los individuos. Esta normatividad inducida se da cuando los vectores que determinan un comportamiento deseado se vuelven aspectos imitables por los individuos; bajo esa estela, el individuo comienza a imitar al dividuo, y no tanto a la inversa, como podría pensarse. Esta necesidad de encajar en el perfil-dividual produce una normalización en la medida en que no se busca "excluir lo que se sale del promedio, sino evitar lo imprevisible, de procurar que todas las personas sean realmente ellas mismas" (Berns y Rouvroy, 2016,p.96). El proceso psicométrico de construcción de perfiles a partir del reconocimiento facial, bajo la lectura de Berns y Rouvroy (2016), no busca solamente clasificar a los individuos en liberales y conservadores, sino generar mecanismos mediante los cuales se pueda ejercer acciones de gobierno. De allí que para estos autores el perfilado está relacionado con un proceso de gubernamentalidad, entendida como los mecanismos de producción y conducción de la conducta.

De acuerdo con Rodríguez (2019), los perfiles-dividuos modifican a los individuos al pasar por un proceso en el cual, a la par que se producen las multiplicaciones, también se generan los individuos. Esto es, a la vez que se perfila, por ejemplo, mediante las facciones del rostro, los individuos se acomodan a los perfiles y, en ese proceso, se producen, se reajustan a esos vectores. En ese proceso se da una "transformación de individuos en datos y recomposición posterior que da como resultado otro individuo relacionado, pero en principio no igual al individuo antes de ser transformado" (Rodríguez, 2019, p. 453). En el caso del perfilamiento mediante el reconocimiento facial, al considerar que los vectores que se toman están relacionados con formas y modos en los que se posa o se mira la cámara, el efecto sobre los individuos de esa medición produce que el perfil-dividuo afecte, recomponga y determine al individuo original. Por tanto, esa producción psicométrica afecta la vida delos individuos en la medida en que, sobre la base de ese perfil-dividuo, se tomen decisiones:

Todas las fragmentaciones, divisiones, multiplicaciones y sustituciones no se refieren a un individuo considerado en su totalidad presente, en su individualidad actual, que es lo que describe la figura del doble, sino más bien a sus potencialidades, a lo que puede ser en función de lo que es y a lo que puedehacer en función de lo que ya está haciendo (Rodríguez, 2019, p. 454).

En síntesis, al determinar las potencialidades (las predicciones de un comportamiento), los estudios psicométricos operan produciendo subjetividades. Bien sea para mejorar la toma de decisiones, que puede cruzar, por caso, lo laboral, financiero o judicial, o bien para que los individuos empiecen a establecer modos en los que puedan encajar en las normalidades establecidas o se ajusten a esos vectores determinados. De allí que las necesidades de encuadrar ayuden a configurar y producir las formas subjetivas que deben, de acuerdo a la norma, adoptarse. 


\section{APUNTES FINALES}

Para Pasquinelli y Joler (May 1, 2020), muchos de los problemas que enfrenta la IA y el aprendizaje automatizado tienen que ver con la producción de sesgos, entre los cuales se encuentran discriminaciones por rasgos raciales, género, etc. Para ellos, se debe distinguir entre sesgos históricos, de datos y algorítmicos. El primero, también denominado sesgo de mundo, refiere al contexto de exclusión existente, al afuera de la programación y del sistema computarizado, en donde se presentan discriminaciones históricas y sociales. Este sesgo apela a contextos de exclusión, que muchas veces son sesgos estructurales que se trasladan al funcionamiento de los sistemas automatizados. Un estudio representativo de esa forma de sesgo ha sido analizado por Benjamin (2019), quien estudió de qué manera las formas del racismo norteamericano se refuerzan, transforman o integran a la programación de sistemas algorítmicos.

El segundo tipo de sesgo señalado, el de datos, supone que las discriminaciones se ponen en circulación cuandolos programadores introducen exclusiones en el momento en el que entrenan a los sistemas de IA. Bajo esa perspectiva, los desarrolladores pueden basarse en taxonomías hegemónicas que conllevan malinterpretaciones de diversidad social o reproducir jerarquías sociales (por ejemplo, creer que una persona de tendencias políticas conservadoras viste bien o alguien liberal tiene el cabello largo). Mientras que el tercer tipo de sesgo es el algorítmico. También conocido como sesgo estadístico, este sesgo está relacionado con el modelo mismo de la programación en el que se basa el aprendizaje automatizado. Así, la cuestión radica en que los algoritmos se consideran como sistemas eficientes en la "compresión de información" y, en ese proceso, se da una sustracción, representación y "pérdida de información" de la realidad. Por tanto, esa sustracción de la realidad mediante aspectos relevantes, efectuado a través de la ponderación de elementos que se consideran significativos o variables que permiten dar cuenta de comportamientos humanos, produce una "pérdida de la diversidad cultural" que afecta directamente a la sociedad (Pasquinelli \& Joler, May 1, 2020).

Teniendo en cuenta este último tipo de sesgo, en el cual el sistema mismo, el procedimiento teórico y metodológico sobre el que se basa la programación de algoritmos, en este artículo se ha intentado mostrar cómo opera esa reducción de la realidad a través de variables sobre las cuales se quiere acomodar, clasificar y predecir comportamientos humanos. Los estudios psicométricos sobre el reconocimiento facial están enfocados en determinar rasgos latentes como las filiaciones políticas a través de pequeñas y pocas características del rostro: posición de la cabeza cuando se posa, gestos, uso de lentes o gafas y corte de cabello. Esta sustracción y abstracción de elementos puede derivar en sesgos sociales que pueden reproducir jerarquías o discriminaciones estructurales. 
Sibien el aprendizaje automatizado ha estado envuelto en su mistificación, una forma de comprensión que privilegia lo oculto, o la caja negra, lo que se procuró fue poner en superficie es una forma de empezar a abrir dicha caja negra y entender los modos en los que se construyen esos sistemas de observación. En otras palabras, entender los modos en los que operan estudios psicométricos basados en IA permite observar el interior de la programación de plataformas -como las redes sociales-o de sistemas biométricos-como el reconocimiento facial-. Esto se pudo observar dado que la psicometría está basada en una recopilación de información, en un procesamiento estadístico enfocado en la programación de algoritmos, que permite elaborar un resultado que tiene unas implicaciones sociales, puesto que están diseñados para una toma de decisiones (por ejemplo, dar o negar un crédito). Así, se buscó desplazar la idea de que la biometría es problemáticas por el hecho de que recopilar información, cuando la realidad es que la producción de herramientas, diseños o modelados se traducen en perfiles utilizables para clasificar y predecir los comportamientos de los individuos. Ese perfilado, como se intentó mostrar, se efectúa con base en variables que permiten inferir rasgos latentes. Con ese soporte, la psicometría plantea que se pueden predecir comportamientos a partir de la posición de los brazos, la edad, los gestos, los rostros. Esos perfiles, construidos bajo esa modalidad, afectan o amenazan con modificar aspectos políticos, económicos, de vigilancia, educativos, en fin, de la sociedad en su conjunto.

\section{REFERENCIAS}

Ballew, C.\&Todorov, A. (2007).Predicting politicalelections from rapid and unreflective facejudgments. Proceedings of the NationalAcademyofSciences, 104, 17948-17953.

Benjamin, R. (2019).Raceaftertechnology:Abolitionist toolsforthenewjim code. Oxford: Social Forces.

Berns, T. \& Rouvroy, A. (2016). Gubernamentalidad algorítmica y perspectivas de emancipación. ¿La disparidad como condición de individuación a través de la relación? Adenda Filosófica, (1), 88-116.

Bishop, C. (2006). Pattern Recognition and Machine Learning. Berlin: Springer.

Brand, S. (1994). How buildings learn: What happens after they're built. New York: Viking-Penguin.

Cao, Q., Shen, L., Xie, W., Parkhi, O. M. \& Zisserman, A. (2018). Vggface2: A dataset for recognising faces across pose and age. In AA.VV., IEEE International Conference on Automatic Face \& Gesture Recognition (pp. 67-74). DOI Bookmark: 10.1109/ FG.2018.00020 
Carpinella, M. \& Johnson, K. (2013). Appearance-based politics: Sex-typed facial cues communicate political party affiliation. Journal of Experimental Social Psychology. 49(1), 156-160. ¡DOI: https://doi.org/10.1016/j.jesp.2012.08.009

Deleuze, G. (1999). Posdata sobre las sociedades de control. En Ferrer, C. (Ed.), El lenguaje libertario. Antología del pensamiento anarquista contemporáneo (pp. 101-109). Buenos Aires: Altamira.

Geisinger, K. (Ed.) (2013). APA Handbook of Testing and Assessment in Psychology. Vol 1. Test Theory and Assessment in Industrial and Organizational Psychology. Washington, DC: American Psychological Association.

Gelman, A. (2013). Bayesian Data Analysis. London: CRC.

Gómez-Barrera, J. (2020). Las huellas digitales del comportamiento humano: gustos, neoliberalismo y algoritmos. MEDIAÇÕES, 25(3). DOI: $10.5433 / 2176-6665.2020 .3 \mathrm{v} 25 \mathrm{n} 3 \mathrm{p} 712$

Hill, K. (June 24, 2020). Wrongfully Accused by an Algorithm. The New York Times. Recuperado de: https://www.nytimes.com/2020/06/24/technology/facialrecognition-arrest.html

Holden, D. (2000). Psycometrics. In Kazdin, A. (Ed.), Encyclopedia of psychology (pp. 417-419). New York: Oxford University Press.

Jones, L. \& Thissen, D. (2007). A History of Overview of Psychometrics. In Rao, C. \& Sinharay, S. (Ed.), Handbook ofStatistics 26. Psychometrics (pp. 1-27). Amsterdam: Elsevier.

Kosinski, M. (2021). Facial recognition technology can expose political orientation from naturalistic facialimages. Nature Research, 11. DOI:https://doi.org/10.1038/ s41598-020-79310-1

Lipton, Z. C. (2016). The Mythos of Model Interpretability: In machine learning, the concept of interpretability is both important and slippery. Queue, 16(3), 31-57.

Markman, A. (1998). Knowledge representation. Mahwah: Erlbaum.

Martínez, A., Hernández, M. \& Hernández, L. (2006). Psicometría. Madrid: Alianza.

Messick, S. (1989). Validity. In Linn, R. (Ed.), Educational measurement (pp. 13-103). New York: American Council on Education/Macmillan.

Mislevy, R. J. \& Gitomer, D. H. (1996). The role of probability-based inference in an intelligent tutoring system. User-Modeling and User-Adapted Interaction, 5, 253-282.

Muñiz, J. (2003). Teoría clásica de los tests. Madrid: Pirámide.

Pasquinelli, M. \& Joler, V.(May 1,2020).TheNooscope Manifested:Artificial Intelligence as Instrument of Knowledge Extractivism. KIM HfG Karlsruhe and Share Lab. Recuperado de: https://nooscope.ai 
Pearl, J. (1988). Probabilistic Reasoning in Intelligent Systems. San Francisco: Morgan Kaufmann.

Redacción MIT Technology Review (1 de marzo de 2021). Ocho predicciones sobre el impacto de la tecnología en nuestra vida en 2021. MIT Technology Review. Recuperado de: https://www.technologyreview.es/s/13212/ocho-prediccionessobre-el-impacto-de-la-tecnologia-en-nuestra-vida-en-2021

Roberts, T. \& Bruce, V.(1988). Feature saliency in judging the sex and familiarity offaces. Perception, 17, 475-581.

Rodríguez,P.(2019).Laspalabrasenlascosas: saber,poderysubjetivaciónentrealgoritmos ybiomoléculas. Buenos Aires: Cactus.

Rule, N. \& Ambady, N. (2008). The face of success: Inferences from Chief Executive Officers' appearance predict company profits. Psychol Sci, 19, 109-111.

Rule, N. \& Ambady, N. (2010). Democrats and Republicans can be differentiated from their faces. PLoS ONE (5). DOI: https://doi.org/10.1371/journal.pone.0008733

Rust, J. \& Golombok, S. (2009). Modern Psychometrics. The Science of Psychological Assessment. London: Routledge.

Rust, J., Kosinski, M. \& Stillwell, D. (2021). Modern Psychometrics: The Science of Psychological Assessment. London: Routledge.

Sampson, E. E. (1981). Cognitive psychology as ideology. American Psychologist, 36, 730-743.

Segalin, C., Celli, F., Polonio, L., Kosinski, M., Stillwell, D., Sebe, D., Cristani, M. \& Lepri, B. (2017). What your Facebook Profile Picture Reveals about your Personality. Proceedings of the 25th ACM international conference on Multimedia, 460-468. DOI: https://doi.org/10.1145/3123266.3123331

Sibley, C. G., Osborne, D. \& Duckitt, J. (2012). Personality and political orientation: Meta-analysis and test of a Threat-Constraint Model. Journal of Research in Personality, 46(6), 664-677.

Stevens, S. (1946). On the theory of scales of measurement. Science, 106, 667-680.

Stewart,J. \& Hafner,R. (1995). Revising explanatorymodelstoaccommodate anomalous genetic phenomena: Problem solving in the "context of discovery". Science and Education. https://doi.org/10.1002/sce.3730790202

Stone, C. \& Yerkes, R. (1920). Army Mental Test. H.Holt.

Suárez, M. (2004). An inferential conception of scientific representation. Philosophy of Science, 71, 767-779. 
Swoyer, C. (1991). Structural representation and surrogative reasoning. Synthese, 87, 449-508.

Wang, Y. \& Kosinski, M. (2018). Deep Neuoral networks are more accurate than humans at detecting sexual orientation from facial images. Journal of Research in Personality, 114(2). DOI: 10.1037/pspa0000098

Zebrowitz, L. (1997). Reading faces: Window to the soul? Boulder: Westview Press.

${ }^{*}$ Contribución: el trabajo fue realizado íntegramente por el autor.

* Nota: el Comité Editorial de la revista aprobó la publicación del artículo.

\section{(cc) BY}

Artículo publicado en acceso abierto bajo la Licencia Creative Commons - Attribution 4.0 International (CC BY 4.0).

\section{IDENTIFICACIÓN DEL AUTOR}

Juan Camilo Gómez-Barrera. Magíster en Comunicación y Cultura, Facultad de Ciencias Sociales, Universidad de Buenos Aires (Argentina). Editor de investigaciones sobre evaluación y medición, Instituto Colombiano para la Evaluación (Colombia). Ha publicado artículos en distintas revistas académicas, entre otros: "Las huellas digitales del comportamiento Humano. Gustos, neoliberalismo yalgoritmos" (2020)y “Del Homo Economicus al Dividuo: del neoliberalismo ala gubernamentalidad algorítmica” (2021). Sus temas de investigación giran en torno a la epistemología producida por lo digital, las afectaciones económicas, políticas y sociales de la tecnología (en especiallas redes sociales) y las implicaciones sociales de la psicometría y la estadística. 
(N) 